\title{
Dynamic Fragmentation Scheme for Rate-Adaptive Wireless LANs
}

\author{
Byung-Seo Kim, Yuguang Fang and Tan F. Wong \\ Department of Electrical and Computer Engineering \\ University of Florida \\ Gainesville. Florida. 3261 I-6130 \\ jsnbs@ufl.edu. fang@ece.ufl.edu, twong@ece.ufl.edu
}

\author{
Younggoo Kwon \\ Central R\&D Center \\ Samsung Electro-Mechanics \\ 314 Maetan 3-dong, Paldal-gu, Suwon, 442-743, Korea \\ younggoo.kwon@samsung.com
}

\begin{abstract}
Many rate-adaptive $\mathrm{MAC}$ protocols have been proposed in the past for wireless LANs over time-varying media to provide efficient channei usage and throughput enhancement. Most of these protocols are receiver-based and employ RTS/CTS collision avoidance handshakes specified in the Distributed Coordination Function (DCF) of the IEEE802.11 MAC. However, these protocols do not consider the possible bursty transmission of fragments in a rate-adaptive fashion. In this paper. a rate-adaptive protocol with a dynamic fragmentation scheme is proposed in order to enhance the throughput by employing bursty fragment transmissions based on the channel condition. Instead of using one fragmentation threshold, multiple thresholds for different data rates are proposed so that more data can be transmitted at higher data rates when the channel is good. In the fragmentation scheme of IEEE802.11, all fragments are generated with equal size at the same time, and all fragments will not change until they are transmitted. In our proposed scheme. whenever the rate for the next transmission is chosen based on the channel condition estimated from the previous fragment transmission, a new fragment is generated using the fragment threshold for the new rate. In this way. the channel can be more effectively utilized to squecze in more bits. This scheme is evaluated under a time-correlated fading channel model in terms of the achieved throughput. Simulation results show that this scheme achieves a much higher throughput than other rateadaptive protocols do.
\end{abstract}

Kelwords: Fragmentation; MAC; Wireless LANs; Rateadaptive MAC protocol

\section{INTRODUCTION}

A typical wireless communication link is time-varying. Adaptive transmission schemes that adjust to varying channel conditions have been proposed in order to enhance the throughput and channel utilization of a wireless link. This is usually achieved by varying the data rate, transmission power, and packet length of the transmission. Rate adaptation is one such adaptive transmission scheme that employs different modulation and coding schemes to adjust the data rate based on channel conditions, such as the Signal-to-Noise Ratio (SNR). The basic concept is that to obtain a target error rate, a highlevel modulation scheme requires a higher SNR than a lowlevel modulation scheme does. The target error rate can be the Bit Error Rate (BER), Symbol Error Rate (SER) or Packet Error Rate (PER) specified by a designer. For receiver-based rate-adaptation schemes, channel estimation and rate selection are done by the receiver. The selected rate is then sent back to

This work was supported in part by US National Science Foundation under grant ANI-0220287 and US Olfice of Research under grant NO00140210554. the sender. It is the Medium Access Control (MAC) protocol that specifies how the receiver can inform the selected rate to the sender and the other nodes.

For centralized networks such as $2.5 \mathrm{G}$ and $3 \mathrm{G}$ cellular networks, rate-adaptation schemes using $\mathrm{MAC}$ have been investigated [1][2][3][4]. However, to our best knowledge, only a few MAC protocols with rate-adaptation have been proposed for wireless LANs. In [6], the Auto Rate Fallback (ARF) protocol is proposed, in which the sender selects the rate based on the packet transmission failure rate. In [7] and [8], the authors use RTS/CTS collision avoidance handshaking to exchange channel information and select a rate accordingly. Specially, in the Receiver-Based AutoRate (RBAR) MAC protocol proposed in [7], channel estimation and rate selection are done by the receiver, and then the selected rate is sent back to the sender in the MAC header of the CTS packet. With this rate-adaptive protocol, the sizes of transmission packets vary and hence all nodes may have different channel access times. Therefore, in the Opportunistic Auto Rate (OAR) protocol proposed in [9], the authors suggest to allow a sender to use a high data rate to transmit more packets in the duration for which a sender has acquired the channel access right.

In IEEE 802.11, when the MAC Service Data Unit (MSDU) generated by the Logical Link Control (LLC) layer is larger than the fragmentation threshold, the MSDU is fragmented into smaller sized MAC Protocol Data Units (MPDUs). For many applications, the size of MSDU is often so large that further fragmentation is necessary. In [10] and [11], the authors develop a scheme to choose the optimal fragment size based on the information about the channel condition, data rate, and goodput. In this scheme, although each MSDU can be fragmented according to the channel information, the sizes of MPDUs remain unchanged during the MSDU transmission. Another fragmentation scheme is proposed in [12]. In this scheme. the fragmentation threshold is halved after each transmission failure occurs during the transmission of the fragment bursts. The objective of this scheme is to avoid possible repetitive interferers without rate adaptation.

In this paper, we propose a new receiver-based MAC protocol based on a dynamic fragmentation scheme. The proposed protocol is similar to the RBAR and OAR, however, instead of allowing the transmission of multiple packets with a high data rate, we allow larger MPDU size to reduce the overheads caused by multiple packets transinission when the 
channel condition is good. In addition, we adapt the fragment size during the transmission of the fragment bursts based on the channel condition. i.e., a fragment is generated from the remaining MSDU only when a fragment is ready for transmission. in contrast to the one-time fragmentation for MSDU used in other protocols (such as IEEE 802.11 MAC). The rest of this paper is organized as follows. Section II reviews the fragmentation process of the IEEE 802.11 MAC protocol and the rate-adaptive schemes. The proposed scheme is presented in Section III. Section IV describes the simulation enviroment under which the performance of the proposed scheme is evaluated. In Section V, conclusions are provided.

\section{I1. BACKGROUND}

\section{Fragmentation in /EEE 802.11}

Fragmentation is the process of dividing a long frame into several shorter frames. Fig. I illustrates the fragmentation process of IEEE 802.11[5]. When MSDU is passed down from the LLC layer, if its size is greater than afragmentationThreshold. it is fragmented into smaller MAC-level frames, namely MPDUs, Fragmentation can improve transmission reliability in the physical layer because the probability of successful transmission increases as the size of the frame decreases. Usually, the MSDU is fragmented into equal-sized MPDUs except for the last one. In addition, the MSDU will not be refragmented further. All fragments are stored in a buffer and wait for transmission. The fragments are sent as independent transmissions, and each of which is separately acknowledged. Once a sender has contended for the medium, it continues to send fragments with Short InterFrame Space (SIFS) gaps between the reception of the Acknowledgment (ACK) packet and the start of the subsequent fragment transmission until either all the fragments of the MSDU have been sent. or an ACK frame is not received. The transmission process of fragments of an MSDU is called a fragment burst. Since the header of each MAC frame contains the information that defines the duration of the next transmission, the nodes that overhear the header update the NAV value for the next fragment transmission.

B. Rate-Adaptive protocol specified in DCF of IEEE 802. 11 MAC

The RBAR MAC protocol proposed in [7] uses RTS/CTS packets to exchange the selected data rate during transmission period of fragments. The receiver using the RTS packet carries out channel estimation and rate selection. The selected rate then fed back to the sender via the CTS packet. The RTS and CTS packets are exchanged at the base rate to make sure that all nodes in the radio range can receive then without error. The RBAR protocol only considers the case that the MSDU size is

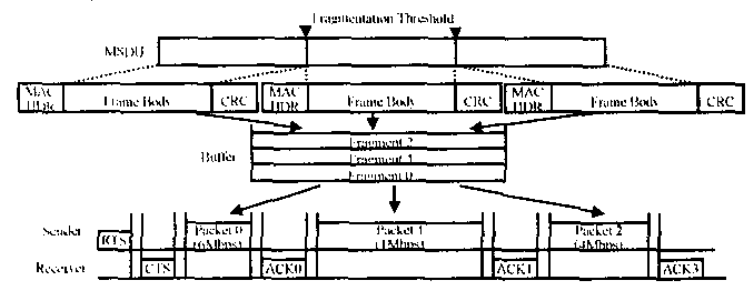

Figure 1. Conventional fragmentation process and the timeline of packet transmission with rate adaptation. less than aFragmentationThreshold, that is, all nodes have only one fragment. With fragment burst transmission and rate adaptation, data and ACK packets also participate in the rate adaptation process in the same way as described above. Since all MPDUs are of the same size, the duration of each packet transmission in the physical layer varies according to the selected rate as shown in Fig. 1. Because of the variable packet transmission duration, a two-step process is proposed in the RBAR protocol for NAV update. The nodes overhearing the CTS packet updates the NAV value with the duration calculated from the selected rate, whereas the nodes overhearing the RTS packet update the NAV value with a tentative duration, which is the duration for transmitting the longest possible packet. When the nodes overhearing the RTS packet hear the data packet, the NAV value is updated to the duration calculated from the rate in the PHY header of the data packet.

\section{PROPOSED PROTOCOL}

We propose a new dynamic fragmentation scheme to enhance the throughput of a wireless LAN system operating under a time-varying environment. The proposed scheme is similar to RBAR and OAR described in Section II, except for the following key changes:

- The durations of all fragment transmissions in the physical layer are set to be the same regardless of the data rate except for the last fragment.

- Different aFragmentationThresholds for different rates are used based on the channel condition.

- A new fragment is generated from the fragmentation process only when the rate is decided for the next fragment transmission.

With a single afragmentationThreshold, the sizes of fragments are equal regardless of the channel condition. Therefore, the channel access times of fragments vary with respect to the selected rate. For example, the channel access time of a fragment at the base rate is longer than that of a fragment at a higher rate. It is generally assumed that the channel is constant during the transmission of a fragment at the base rate. Thus, more data packets can in fact be transmitted when a higher rate is used in the same duration provided that the SNR is high enough to support the higher rate. Based on this observation, the OAR protocol in [9] proposes a multipacket transmission scheme. However, multi-packet transmission has a higher overhead because of additional MAC headers, PHY headers, preambles of data and ACK packets, and SIFS idle times. To overcome the shortcoming of multipacket transmission, we fix the duration of all PHY packet transmission durations except for the last fragment. To better understand the mechanism, we show the protocol timelines for the RBAR scheme and our dynamic fragmentation scheme in Fig. 2. To generate packets with the same duration, the number of bits in a packet (fragment) should be varied based on the selected rate. Thus, it is necessary to have different aFragmentationThresholds for different data rates. When the sender receives the selected rate from the receiver, the next fragment is then generated from the fragmentation process according to the aFragmentationThreshold for that rate. 
In the fragmentation process of IEEE 802.11, MSDU is fragmented into equal-sized fragments, and the fragments remain unchanged until all fragments in the burst are transmitted. If the channel quality is constant during the transmission of the fragment burst, the target PER can be met. However. this scenario is not guaranteed in a wireless LAN. Because of the time-varying nature of a wireless channel, each fragment experiences different channel condition. To achieve the target PER. both the data rate and the fragment size should vary' according to the changing channel condition. Moreover, to better match the varying channel, instead of generating all fragments before transmitting the first fragment, each fragment should be generated at each time when the rate is chosen for the next transmission. As a result, fragments in a burst should not be of the same size. Fig. 3 illustrates the process of the proposed dynamic fragmentation scheme. When the transmission of a fragment fails, the size of the retransmitted fragment may not be the same as that of the originally transmitted fragment since the channel condition may have changed. When the fragment number of the most recently received fragment is the same as that of the already received fragment. the receiver discards the old fragment. Hence, the MSDU size is reduced only when a fragment is transmitted successfully, that is. the sender receives the ACK packet from the recejver.

In the proposed dynamic fragmentation scheme, the NAV update process is simpler than that in the RBAR protocol as described in Section II. Fig. 4 explains the NAV update process in the proposed protocol. Since the durations of all fragments in a fragment burst, except for the last fragment, are the same resardless of the data rate, an overhearing node can update the NAV value to the predefined duration when the More Fragments bit in the MAC header is set to 1. For the last fragment whose More Fragments bit is set to 0, the two-step process for NAV update proposed in the RBAR is applied. At first, an overhearing node updates the NAV value with the duration of the normal packet. This is called a tentative update as shown in Fig. 4. When the last fragment and ACK packet are received, the NAV value is changed to the duration value in the MAC header since the duration values of the MAC headers in the last fragment and the ACK packet indicate the duration of the current transmission.

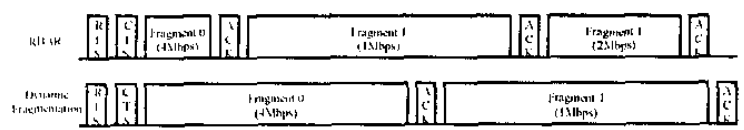

Figure 2. Timelines for RBAR and our dynamic fragmentation.

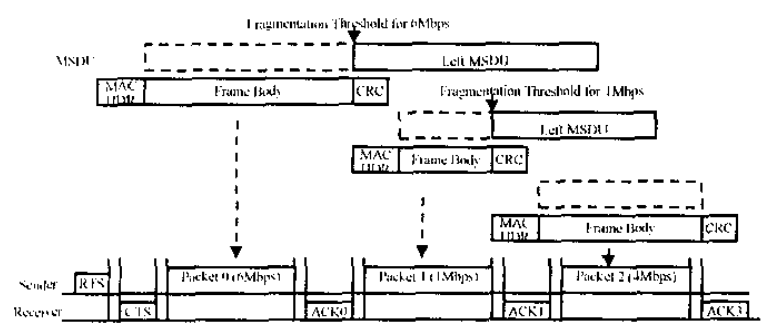

Figure 3.

Dynamic fragmentation process and timeline of packet

transmission

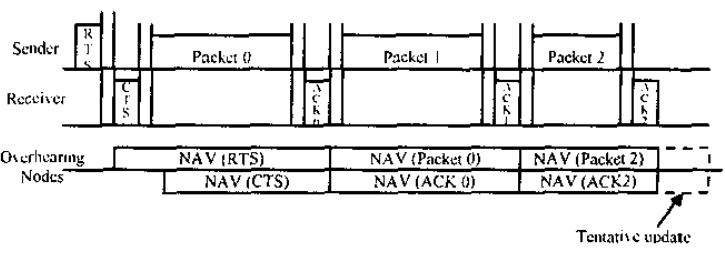

Figure 4. NAV update process in our proposed protocol

\section{PERFORMANCE EVALUATION}

\section{A. Simulation Setting}

We assume that the MSDU size in any node is always larger than the aFragmentationThreshold at the base rate. Thus at this rate, all fragment burst have more than one fragment. We assume that the MSDU size is uniformly distributed over the range from 800 bytes to 8000 bytes at each node. In addition, we assume that all nodes are uniformly distributed in space and within the radio range of each other. Hence the hidden terminal problem is not considered here. The maximum distance between any two nodes is limited to 107 meters based on the indoor environment suggested in $[15]^{2}$. For simplicity, we assume that the headers of all types of packets are always reliably received. Since the control packets such as RTS, CTS and ACK packets are much shorter than data packets, no transmission failure of these packets are considered for simplicity. The parameters used in this simulation study are shown in Table 1. The choices of these parameters are based on the IEEE 802.1 lb DSSS standards except for the MAC header, which is modified from RBAR.

To succinctly demonstrate the ability of the proposed protocol to adapt to the changing channel condition, we assume that the system adapts the data rate by properly choosing one from a set of modulation schemes according to the channel condition. The set of modulation schemes used in this simulation study are BPSK, QPSK, 16QAM, and 64QAM. For simplicity, we ignore other common physical layer components such as error correct coding. A modulation scheme from the above set is chosen so that a target PER can be achieved at the current channel SNR level. Assuming that the symbol errors within a packet are independent, the packet error rate (PER) is related to the SER by

$$
P E R=1-(1-S E R)^{N}
$$

where $N$ is the number of symbols in a packet, which is fixed by the frame duration. We set the target PER to $8 \%$ according to the [EEE 802.11 standard [5]. The number of symbols in a packet except for the last fragment is set to 6400 (cf. [16]). We note that the frame duration is less than the coherence time of the channel for the case that the maximum node speed is $2.5 \mathrm{~m} / \mathrm{s}$. Thus the channel condition is essentially constant within a frame. With these choices of parameters, the target SER is $1.3 \times 10^{-5}$ from (1). To maintain a constant frame duration, the fragmentation threshold at rate $\mathrm{R}$ should be set to

$$
\text { Threshold }_{n}=\text { Threshold }_{B} \cdot R
$$


where Threshold is aFragmentationThreshold at the base rate and its unit is bit. By consulting the SER performance curves in Fig. 5. the SNR ranges for corresponding modulation schemes that the target SER is satisfied are shown in Table Il. Table II also shows the SNR ranges for the conventional fragmentation scheme described in Section II. We note that these ranges are different from those of the dynamic fragmentation scheme because the packet lengths for the two schemes are different so that the different SNR ranges need to be applied to meet the same target SER requirement.

We evaluate the performance of the proposed scheme in a time-correlated fading channel. Under this model, the SNR of the received signal is

$$
S N R=\alpha^{2} \cdot S N R
$$

where $S_{N R}$, is the long-term signal-to-noise ratio determined by the log-distance path loss between the transmitter and receiver [14] and $\alpha$ is a Ricean fading gain. We set the path loss exponent to 4 in the log-distance path loss model. This value is commonly used for an indoor environment. The fading gain $\alpha$ is generated according to the modified Clarke and Gans fading model [13]. For simplicity, we assume that a node can perfectly predict the channel condition for the next fragment transmission. Most of the simulation parameters, e.g. the output power, the antenna gain, the maximum distance and so on, are drawn from the data sheet of the Cisco 350 client adapter [15]. All simulations were performed for the duration of 600 seconds simulation time.

We compare the throughput achieved by the following three different configurations:

- Case 1: rate-adaptive protocol with the proposed dynamic fragmentation scheme;

- Case 2: rate-adaptive protocol with the conventional fragmentation scheme: and

- Case 3: non rate-adaptive protocol with $2 \mathrm{Mbps}$ fixed data rate

\section{B. Impact of Ricean parameter}

Fig. 6 shows the performance of the three configurations described above under different fading environments with 10 nodes and $2 \mathrm{~m} / \mathrm{s}$ node speed. The Ricean parameter, $\mathrm{K}$, repre-

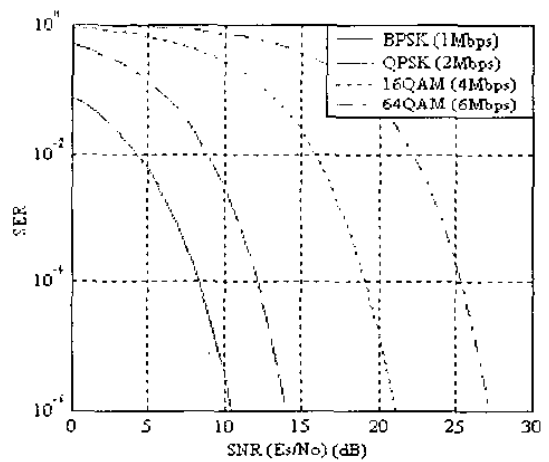

Figure 5. Symbol error rates of BPSK, QPSK, 16QAM, and 64QAM
TABLE I. SIMULATION PARAMETERS

\begin{tabular}{|l|l|}
\hline \multicolumn{1}{|c|}{ Parameter } & \multicolumn{1}{c|}{ Value } \\
\hline SIFS Time & 10 us \\
\hline DIFS Time & 50 us \\
\hline Slot Time & 20 us \\
\hline MAC Header & 304 bits \\
\hline PHY Header & 48 bits \\
\hline Preanble & 144 us \\
\hline ACK frame length & 112 bits \\
\hline RTS frame length & 160 bits \\
\hline C'TS frame length & 112 bits \\
\hline
\end{tabular}

TABLE II. RATE SEL.ECTION CRITERION

\begin{tabular}{|c|c|c|}
\hline \multirow{2}{*}{ Rate (Modulation) } & \multicolumn{2}{|c|}{ SNR Range $(\mathrm{dB})$} \\
\cline { 2 - 3 } & $\begin{array}{c}\text { Dynamic } \\
\text { Fragmentation }\end{array}$ & $\begin{array}{c}\text { Comventional } \\
\text { Fragmentation }\end{array}$ \\
\hline 1Mbps (BPSK) & SNR $<13.08$ & SNR $<12.78$ \\
\hline 2Mbps (QPSK) & $13.08 \leq \mathrm{SNR}<20.07$ & $12.78 \leq \mathrm{SNR}<19.46$ \\
\hline 4Mbps (16QAM) & $20.07 \leq \mathrm{SNR}<26.31$ & $19.46 \leq \mathrm{SNR}<25.5$ \\
\hline 6Mbps (64QAM) & $26.31 \leq \mathrm{SNR}$ & $25.5 \leq \mathrm{SNR}$ \\
\hline
\end{tabular}

sents the strength of the line of the sight component of the received signal. For $\mathrm{K}=0$, the channel has no line-of-sight (LOS) component. This worst-case scenario is usually referred to as Rayleigh fading. As $\mathrm{K}$ increases, the strength of the LOS component increases. Therefore, the performances of the three configurations improve with increasing $\mathrm{K}$. From Fig. 6, we observe that the throughput of employing the dynamic fragmentation scheme is $22.4 \%$ higher than that of not using the dynamic fragmentation scheme.

\section{Impact of node speed}

We vary the speed of nodes, but assume that no nodes are out of the radio range. As the speed of the nodes increases, the coherence time of the channel reduces, that is, the channel changes faster. Fig. 7 shows the performance of the three configurations for 6 different speeds ranging from $0.5 \mathrm{~m} / \mathrm{s}$ to $2.5 \mathrm{~m} / \mathrm{s}$. The number of nodes is 10 and the Ricean parameter $\mathrm{K}$ $=8$. As shown in the figure, the throughput is nearly independent of the node speed since the duration of the packet is less than the coherence time even at the maximum node speed of $2.5 \mathrm{~m} / \mathrm{s}$. From Fig. 7, we observe that the throughput of

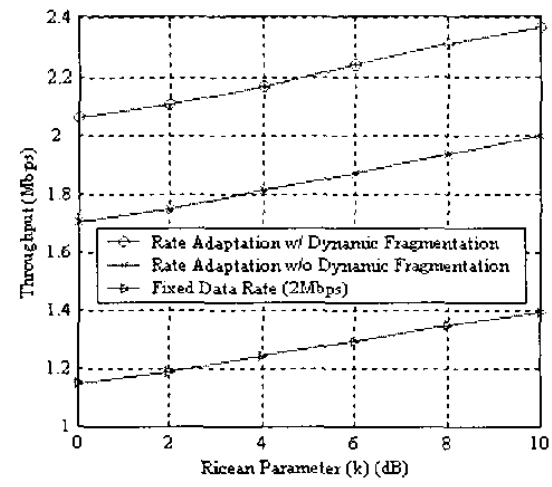

Figure 6. Throughput as a function of Ricean parameter, $\mathrm{K}$ 
employing the dynamic fragmentation scheme is $22.4 \%$ higher than that of not using the dynamic fragmentation scheme.

\section{Impact of number of nodes}

Fig. 8 shows the throughput performance of the three configurations with Ricean parameter $\mathrm{K}=8$ and $2 \mathrm{~m} / \mathrm{s}$ node speed as the number of nodes increases from 10 to 90 . From Fig. 8, we observe that the throughput of employing the dynamic fragmentation scheme is $19.8 \%$ higher than that of not using the dynamic fragmentation scheme. Moreover, we observe that increasing the number of nodes from 10 to 90 causes a $2.4 \%$ degradation in throughput. This can be explained by the fact that no hidden nodes are assumed in the network. Without hidden nodes, the idle time caused by one collision is the sum of the RTS/CTS transmission time, one SIFS, and one DIFS. This idle time duration is small compared to the lost time caused by packet errors. In addition, in the fragment burst transmission, the channel access time of a node is longer than that of a single packet transmission because once the node gains channel access, it transmits several fragments without any further contention. Thus, the effect of increasing collisions due to the larger number of nodes on the throughput is small.

\section{CONCLUSION}

In this paper, we propose a new rate-adaptive MAC protocol with dynamic fragmentation. The major innovation is the use of multiple fragmentation thresholds for different rates to generate a new fragment from the MSDU only after the rate for the next transmission is selected. With this scheme, the node with good channel quality is allowed to transmit more data than the one with bad channel quality over the same amount of channel access time. In addition, the use of constant fragment transmission duration in the physical layer simplifies the process of NAV update in a rate-adaptive system. Simulation results show that the proposed dynamic fragmentation scheme gives a $22 \%$ throughput gain over the conventional fragmentation scheme in IEEE 802.11.

\section{REFERENCES}

11) N. Morinaga. M. Nakagawa, and R. Kohno. "New concepts and lechnologies for achieving highly reliable and high-capacity multimedia wireless communications systenis," IEEE Communications Magazine. pp. 34-40. January 1997.

[2] T. Ikeda, S. Sampei, and N. Morinaga. "TDMA-based adaptive modulation with dynamic channel assignment for high-capacity communication systems." IEEE Trans. Vehicular Technology, vol. 49. pp. 404-412, March 2000.

[3] J. C. -1 . Chuang, and N. R. Sollenberger. "Spectrim resource allocation for wireless packet access with application to advanced cellular internet service." IEEE Journal on Selected Area in Commenication, vol. 16. pp. 820-829. August 1998

[4] S.F. Sheu. Y.H. l.ee. and M.-H. Chen. "providing multiple data rate in infrastructure wireless networks," Proc. IEEE GLOBECOM '0l, vol. 3, pp. 1908-1912, 2001.

151 IHEL: 802.11 WG. Part 11: Wirciess LAN Medium Access Control (MAC) and Physical Layer ( $\mathrm{PHY}$ ) specilications, Standard, IEEE. 1999.

[6] A. Kamerman and 1. Monteban, "WaveLAN-II: a high-pertormance wireless LAN for the unlicensed band." Bell Labs Technical Journal, pp. 118-133. Summer 1997

[7] G. Fiolland. N. Vaidya, and P. Bal11, "A rate-adaptive MAC Protocol for wireless networks." Proc. ACM MOBICOM 01, pp. 236-251, July 2001 .

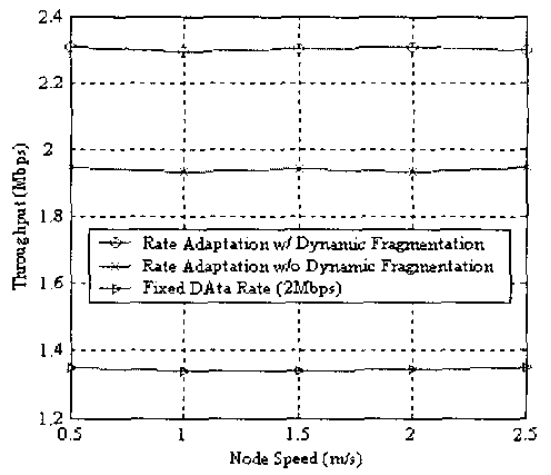

Figure 7. Throughput as a function of specd of nodes

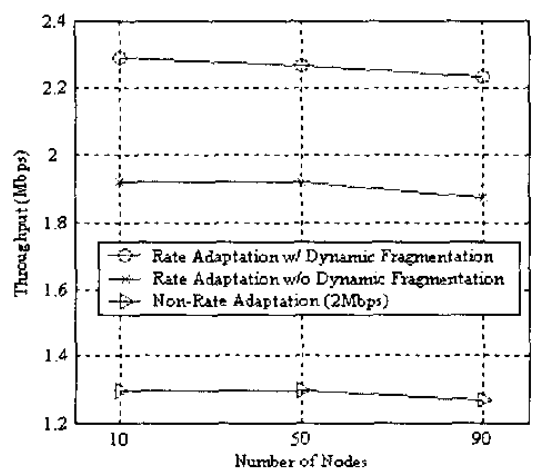

Figure 8. Throughput as a function of the number of nodes

[8] H.-H. Liu. J.-L. C. Wu, and W.-Y. Chen, "New frame based networh allocation vector for $802.1 \mathrm{lb}$ multirate wireless LANs," IEE Proc. Communication, vol. 149, pp 147-151, June 2002.

[9] Sadeghi, V. Kanodia, A. Sabharwal, and E. Knightly, "Oppotunistic media access for multirate ad hoc networks," Proc. ACM MOBICOM" 02, September 2002.

[10] D. Qiao, and S. Choi. "Googput enhancement of IEEE 802.11 a wireless LAN via link adaptation," Proc. IEEE ICC'01, vol.7, pp. 1995-2000. June 2001 .

[ii] D. Qiao, S. Choi, and K. G. Shin, "Goodput analysis and link adaptation for IEEE 802.1! a wireless LANs," IEEE Trans. Mobile Computing, vol. 1. pp. 278-291. December 2002.

[12] J. Tourrihes, "Fragment adaptive reduction: coping with various interferers in radio unlicensed bands," Proc. IEEE ICC'01. vol. I, pp. 239-244, 2001.

[13] R. J. Punnoose, P. V. Nikitin, and D. D. Stancil, "Efficient simulation of riceas fading within a packet simulator," Proc. IEEE VTC'00. pp.764. 767. 2000.

[14] T. S. Rappaport. "Wireless communications: principles and practices," Prentice Hall. 1996.

[15] Cisco Aironet 350 Series Client Adaptors Data Sheet. 2002. http:/www.cisco.com/warp/public/cc/pd/witc/ao350ap/prodlit/a350c_ds. htm.

[16] B. P. Crow, I. Widjaja, J. G. Kim, and P. T. Sakai, "IEEE 802.11 wireless local area networks," IEEE Communication Magazine. pp. 116 126. September 1997. 